\title{
Research on Near Space Plasma Vacuum Environmental Simulation Technology
}

\author{
Fu Chunyu', Dong Yize ${ }^{2}$ \\ ${ }^{1}$ Beijing Insititute of Spacecraft Environment Engineering, Beijing 100094, China \\ ${ }^{2}$ Space Star technology co., LTD, Beijing 100095, China
}

\begin{abstract}
The operation reliability of spacecraft in orbitis affected by the interaction with space plasma, research on the space enviroment by advanced testing method is important. It is costly to carry out research and analysis with space flight. Therefore, tests using ground vacuum environment simulation system are of significance. This paper proposed the structure design, simulation analysis and numerical calculation methods for the three subsystems of plasma environment simulation system, including vacuum vessel, vacuum acquisition and vacuum measurement and control. The simulation results show that the maximum stress of the vacuum vessel is $113.2 \mathrm{MPa}$, and the maximum deformation is $0.59 \mathrm{~mm}$, at the same time, vacuum technology index, structural stability and human-machine interaction performance of the system can meet the experimental research requirement.
\end{abstract}

\section{Introduction}

The traditional research method of space plasma is that launching a deep space rocket or satellite to obtain the data parameters of space plasma. The space testing instruments are expensive and non-reusable, which is difficult to accurately research many microphysical processes. Because of that the laboratory plasma has the characteristics of easily repetition, accurate diagnosis, and low $\operatorname{cost}^{[1-3]}$, it's a new method and a development trend that using laboratory plasma to simulate space physical processes ${ }^{[4-7]}$. The large proximity space plasma vacuum environment imitation system is an important part of the national major science and technology infrastructure "space environment ground simulation device (SESRI)", it's the main part of the space plasma environment simulation and research system.

This paper describes a large-scale near space plasma vacuum environment simulation technology. It is used to provide the vacuum testing environment for space plasma environment simulator, at the same time, it also provide testing environment for the key components such as antenna groups, target analog components, magnet coils, plasma sources, as well as installed base and interfaces for other units. The system is not only a large vacuum system but also a complex mechatronic system. The most basic requirements are system vacuum specifications, structural stability and high reliability ${ }^{[8]}$. The system consists of negative pressure vessel system, vacuum vessel system, and control system. The main functional indicators of this system include:
-The effective space of vacuum vessel: diameter is $\Phi 5000 \mathrm{~mm}$ column length is $6000 \mathrm{~mm}$;

-Extreme vacuum degree ${ }^{[9,10]}: \leq 10 P a$ (This indicator will be reached within 6 hours from atmospheric pressure);

-Operating vacuum degree: when the vessel extracted air achieves the extreme vacuum degree, it filled with argon can continuously adjust the vacuum degree. When the air inflow is in the range of 20SLM $\square 0$ SLM , the working pressure can be achieved the scope of $100 P a \square 1000 P a$, and the control accuracy should surpass $\pm 5 \%$;

-Total leak rate of vacuum system: sum of leakage rate at each leak points are $\leq 1 \times 10^{-5} \mathrm{PaL} / \mathrm{s}$.

\section{Vacuum vessel system}

\subsection{Main structure}

The structure of near space plasma environment vacuum vessel is horizontal cylindrical, One side of the vessel is a gate, which uses electric switch. It is designed according to pressure vessel design standards: it uses material is 304 stainless steel, its inner surface is polished, the roughness is no greater than $1.6 \mu \mathrm{m}$, the length of the straight section is $6000 \mathrm{~mm}$, the inner diameter is $95000 \mathrm{~mm}$, its total length is about $8454 \mathrm{~mm}$. Its structure is shown in figure 1 . 

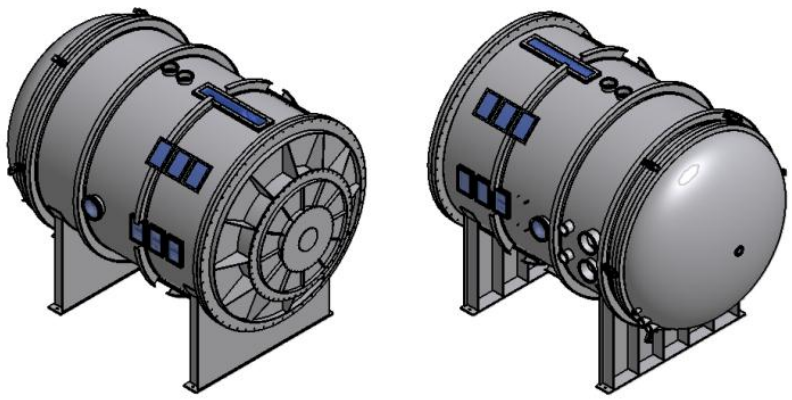

Figure 1. The structure diagram of near space plasma environment vacuum vessel

\subsection{Finite element analysis of container system structure}

Finite element analysis of container system structure is a calculation of intensity proofread and structural stability about vacuum vessel in all kinds of working conditions. It also analyzes the accuracy and verifies the convergence of the calculation results. According to the calculation results, it will give suggestions about improvement and optimization of vacuum vessel's structure.

The 3D model of vacuum vessel is geometric cleaned, and meshed under the physical structure, which is shown in figure 2.

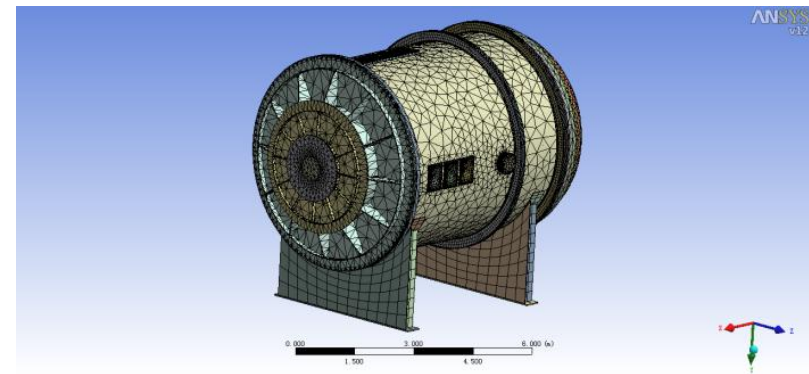

Figure 2. Grid diagram of the near space container

The physical structure is divided into physical units. Added material properties are that the observation window is quartz glass and the rest is stainless steel as required.

The boundary conditions of the model are set as: six DOFs of one saddle are constrained, vertical direction of the other one is constrained. Experimenters submit the analysis of this model, and observe local stress/displacement or distributed stress/ larger displacement area's stress/displacement analysis chart and maximum stress/displacement value.

\subsubsection{Calculation results of finite element analysis}

Overall stress distribution and the location of the maximum stress is shown in figure 3. The maximum stress is $113.2 \mathrm{MPa}$. Areas with higher stress are mainly concentrated at the transfer flange of the vacuum vessel flat end cap.

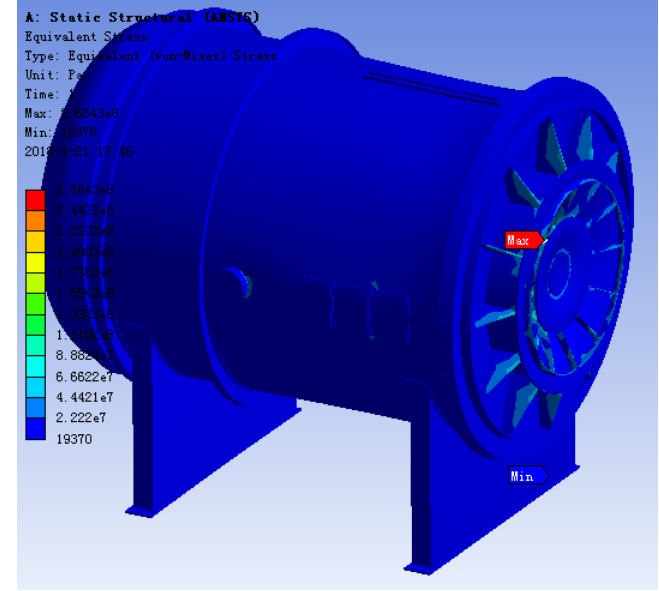

Figure 3. Overall stress distribution and maximum stress position of the vacuum vessel

Overall displacement distribution and maximum displacement position of the vacuum vessel is shown in figure 4. The maximum deformation displacement is $2.85 \mathrm{~mm}$, which appears in the middle of the flat end cap subassembly.

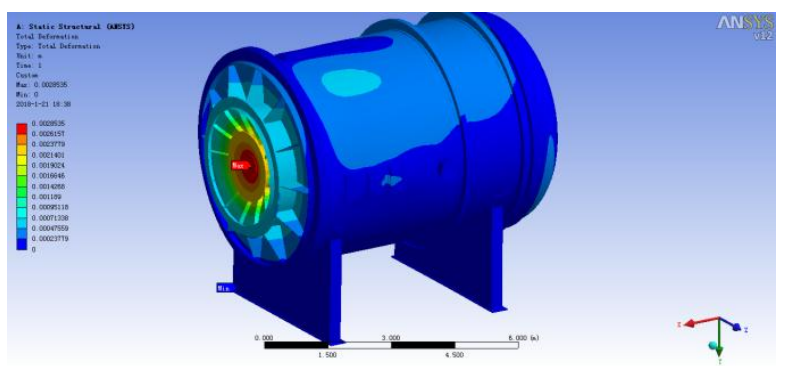

Figure 4. Overall displacement distribution and maximum displacement position of the vacuum vessel

Local deformation extracted from the straight section is shown in figure 5. It can be seen that the structurally deformed area is mainly located on one side of the vessel wall at flange opening, the maximum structural deformation displacement of this area is $0.59 \mathrm{~mm}$.

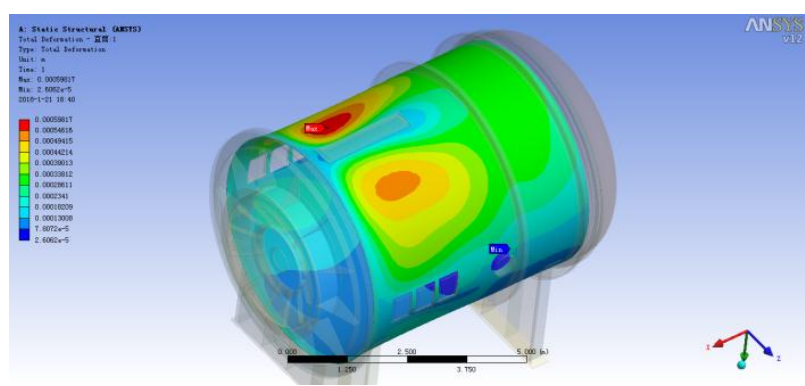

Figure 5. Displacement of the straight section

\subsubsection{Results and discussion}

(1) The vacuum vessel has a low overall stress level. The maximum stress is $113.2 \mathrm{MPa}$, which is less than the allowable stress of stainless steel. The structural strength of the vacuum vessel meets requirements. 
(2) The local deformation of the straight section shows that the maximum structural deformation displacement is $0.59 \mathrm{~mm}$. Therefore, the offset of the antenna position caused by the vacuum deformation of near space vessel does not exceed $0.59 \mathrm{~mm}$, which meets requirements of the mission.

(3) It can be seen from the finite element calculation results that all of the strength and deformation of the container system meet requirements, and the container will be stable.

\section{Vacuum system}

\subsection{Function and composition}

The near space plasma vacuum system is mainly used to provide a vacuum acquired capacity, which can meet the requirements of clean vacuum quality and the device operation. The system schematic is shown in figure 6 .

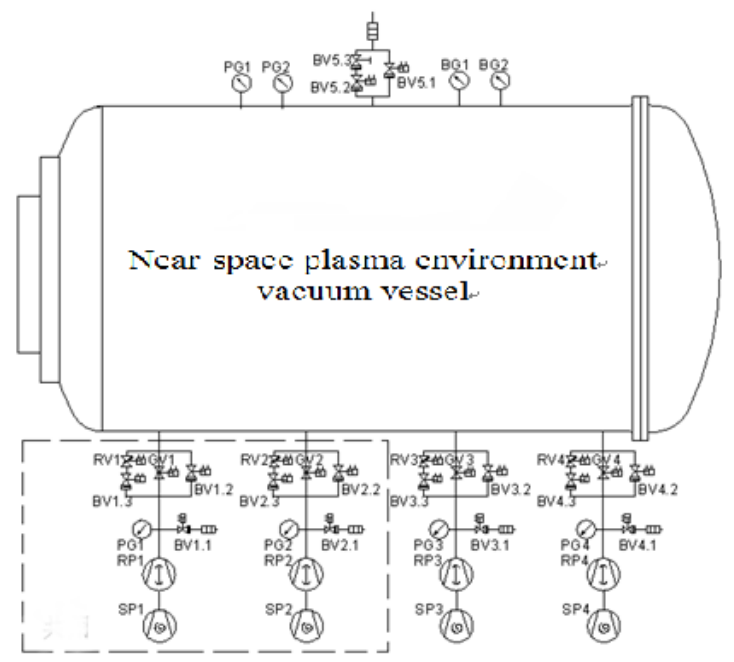

Figure 6. The composition diagram of the vacuum vessel system

\subsection{Design and calculation of the vacuum system}

(1) The pumping speed required for ultimate vacuum

The pumping speed required for the vacuum system to reach the required limit or working vacuum is determined by the following formula:

$$
P_{g}=P_{0}+\frac{Q}{S_{e f f}}+\frac{Q_{1}}{S_{e f f}}
$$

$P_{g}$ - The ultimate vacuum that can be achieved by the pod, $P a$;

$P_{0}$ - The ultimate vacuum of vacuum pump, $P a$;

$Q_{0}$ - The gas load of the vacuum vessel (no-load) after a certain period of evacuation (including leakage $Q_{L}$, material surface leak $\left.Q e\right), P a L / s$;

$Q_{1}$ - The gas load of vacuum vessel;
$S_{\text {eff }}$-Effective pumping speed near the exhaust port of the vacuum vessel, $L / s$.

In formula (1), the ultimate vacuum of the vacuum vessel is always lower than the ultimate vacuum of a vacuum pumping unit, which is $P_{0} \leq P_{g}$. Under certain condition of effective pumping speed, the ultimate vacuum of the vacuum vessel with no-load is proportional to the air leakage and exhalation of the vacuum vessel.

According to the size of the vacuum vessel and related data of other subsystem, this paper calculates the total deflation load released from the surface of the vacuum vessel. The specific data is shown in table $1 \& 2$.

Table 1. The calculated value of the gas outlet from the vacuum vessel

\begin{tabular}{|c|c|c|c|c|c|}
\hline \multirow{2}{*}{\multicolumn{2}{|c|}{ Outgassing meterial }} & \multicolumn{4}{|c|}{ Pumping time } \\
\hline & & 1 & 2 & 5 & 10 \\
\hline $\begin{array}{c}\text { Inner } \\
\text { surface } \\
\text { area of }\end{array}$ & $\begin{array}{l}\text { Outgassing } \\
\text { rate } \\
\mathrm{PaL} / \mathrm{scm}^{2}\end{array}$ & $2.8 \times 10^{-7}$ & $1.73 \times 10^{-7}$ & $9.33 \times 10^{-8}$ & $5.33 \times 10^{-8}$ \\
\hline $\begin{array}{c}\text { vacuum } \\
\text { vessel } \\
\left(180 m^{2}\right)\end{array}$ & $\begin{array}{c}\text { Gas } \\
\text { volume } \\
P a L / s\end{array}$ & 0.50 & 0.31 & 0.18 & 0.10 \\
\hline
\end{tabular}

Table 2. The calculated value of the gas outlet inside the vacuum vessel

\begin{tabular}{|c|c|c|}
\hline Deflating object & $\begin{array}{c}\text { Outgassing amount in } \\
\text { unit time } / \mathrm{Pam}^{3} \mathrm{~s}^{-1}\end{array}$ & Quantity \\
\hline $\begin{array}{c}\text { Composite } \\
\text { absorber }\end{array}$ & $\begin{array}{c}\text { Outgassing amount of } \\
\text { individualmodule } \\
2 \times 10^{-1}\end{array}$ & $\begin{array}{c}\text { Not less than } \\
400\end{array}$ \\
\hline
\end{tabular}

The leakage rate must be limited to ensure that the air leakage does not affect the ultimate vacuum with no load, The leakage rate of vacuum vessel equipment should be 10 times lower than the outgassing rate. Because the container's flanges and connectors easily lead to leakage, the leakage rate of these components accounts for a large part of the allowable leakage rate. The weld of the container will not be dismantled. According to technical requirements, the total leakage rate of the vacuum system is $\leq 1 \times 10^{-5} \mathrm{PaL} / \mathrm{s}$.

Therefore, when the vacuum vessel is unloaded (there is no device inside), the internal gas load is determined as $5.0 \mathrm{~Pa} \cdot \mathrm{L} / \mathrm{s}$, the amount of air release in the vacuum vessel,which is assembled the microwave darkroom and all supporting components, is about $8.0 \times 10^{4} \mathrm{PaL} / \mathrm{s}$. Replace the amount of vent gas with the required ultimate vacuum into formula (1), it can be seen that the required pumping speed for the vacuum obtaining system needs to be not less than $8000 \mathrm{~L} / \mathrm{s}$ (approximately $28800 \mathrm{~m}^{3} / \mathrm{h}$ ). 

time

(2) Calculating pumping speed required for pumping

Vacuum system also requires that the vacuum vessel pressure $\leq 10 \mathrm{~Pa}$ in 4 hours (after pumping from atmospheric pressure); vacuum vessel system satisfies the pumping time requirements of vacuum storage compartment. Calculating the pumping speed according to the following formula:

$$
S e=2.3 \frac{V}{t} K \times L g \frac{P_{1}}{P_{2}}
$$

$t$ - Pumping time, $h$;

Se - - Effective pumping speed of dry pump, $m^{3} / h$;

$V$-Container volume, $m^{3}$;

$P_{1}$ - Pressure at the start of pumping, $\mathrm{Pa}$;

$P_{2}$ - Pressure after $t$ time, $P a$;

$K-$ Correction factor.

Above all, to satisfy the vacuum indicator needed for vacuum vessel of near space plasma vacuum environmental simulation subsystem, the minimum pumping speed required for the vacuum system is not less than $8000 \mathrm{~L} / \mathrm{s}$ (approximately $28800 \mathrm{~m}^{3} / \mathrm{h}$ ).

(3) Review and recalculate of the vacuum system

According to the preliminary layout position of the roughing unit, it can be assumed that the length of the roughing main pipeline is estimated to be within about $10 \mathrm{~m}$. The conductance of rough pipe can be calculated by the following formula for viscous flow and long pipe flow conductance:

$$
U=1.34 \times 10^{3} \frac{d^{4}}{L} \bar{P}
$$

$U-$ Conduction of the main pumping pipe, $\mathrm{m}^{3} / \mathrm{s}$;

$d$ - The inside diameter of the main pumping pipe, $m$;

$L-$ The length of the main pumping pipe, $m$;

$\bar{P}$ - - Average pressure in the pipeline, $P a$.

Review and recalculate the capacity of the selected vacuum unit, according to formula (1), rough pumping unit can pump the vacuum vessel to $\leq 10 P a$, which meet the requirements of ultimate vacuum. Evaluate the extraction time according to formula (2), vacuum system draws container to desired pressure within 1.5 hours, which meet the time requirements for pumping.

\section{Control system}

\subsection{System structure}

The control system of the near space plasma vacuum system adopts local control and remote control modes, it is mainly used to control and monitoring the vacuum vessel system and accessory equipment. The structure is shown in figure 7.

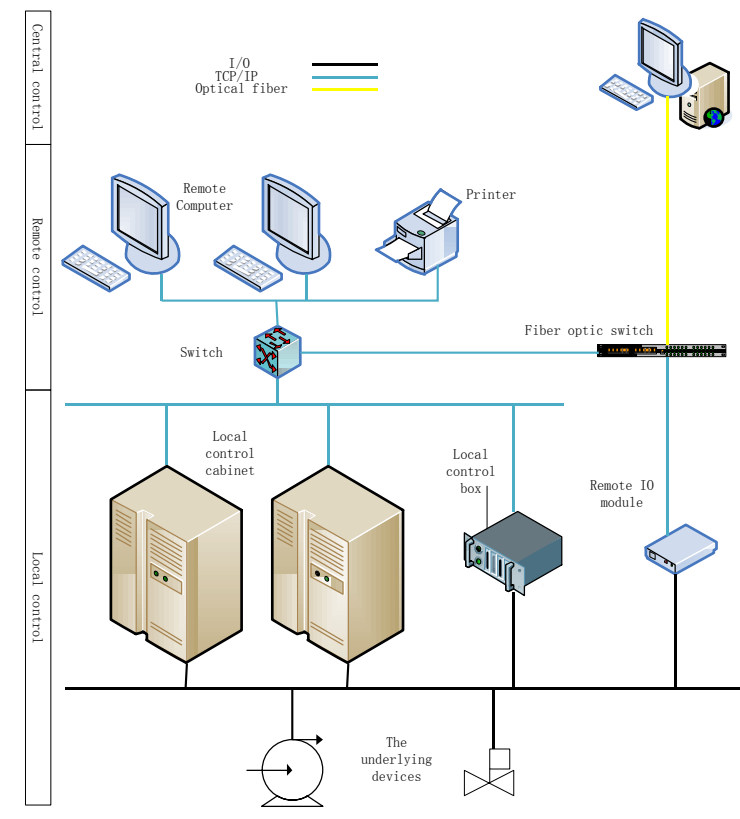

Figure 7. The structure of the control system

The local control system selects the distributed control architecture controlled by S7-300 controller, it is integrated into the local control cabinet. The configuration software can control all controllable equipment and monitor the measurable parameter. The remote control adopts the fusion mode composed by a scheme based on industrial control universal configuration software and a scheme based on universal programming language self-developed software.

\section{System software}

The software design of control system includes the design of the PLC bottom layer program and the design of the configuration software. The overall structure of the software is shown in figure 8 .

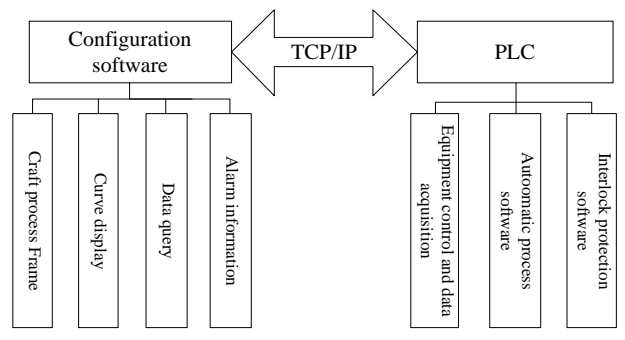

Figure 8. General structure of the software

The PLC bottom program is developed with STEP7, it implements the following man-machine interface:

(1) Craft process frame: According to the vacuum system process design, the craft process frame is established, and the operation status and real-time date of each device can be viewed on screen. In addition, devices, 
which have accessible control, can be controlled by buttons on screen. Technological flow chart is shown in figure 9 .

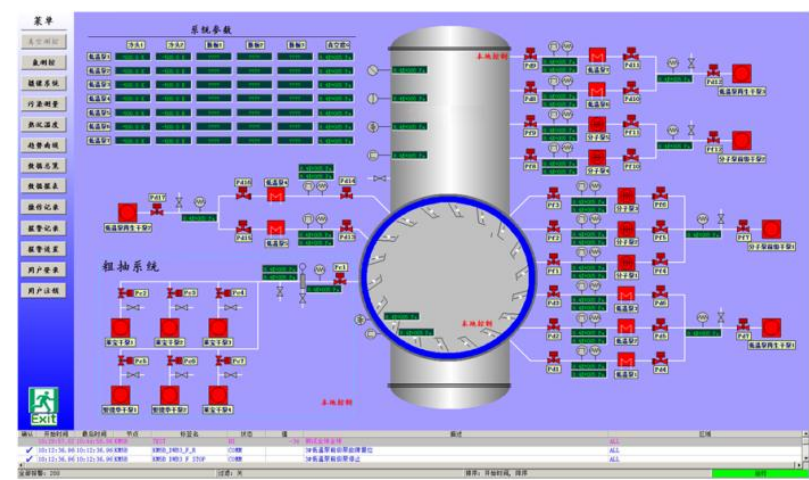

Figure 9. Technological flow chart

(2) Curve display: All the collected real-time date is displayed in the form of curve. Multiple curves can be displayed simultaneously or separately, and history curves can be queried. The schematic of curve display is shown in figure 10 .

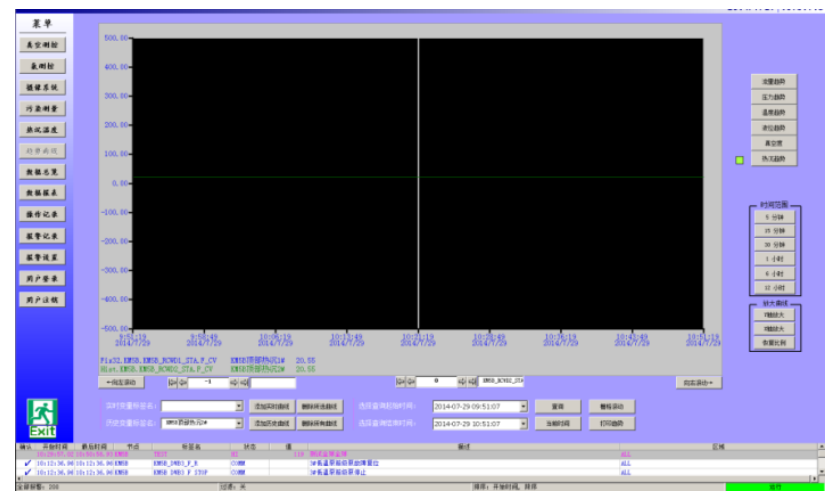

Figure 10. The schematic of curve display

(3) Date query: Establishing an independent data query can query the historical operating state and the historical date of the system, including operation logs and key historical date. The schematic of data query is shown in figure 11.

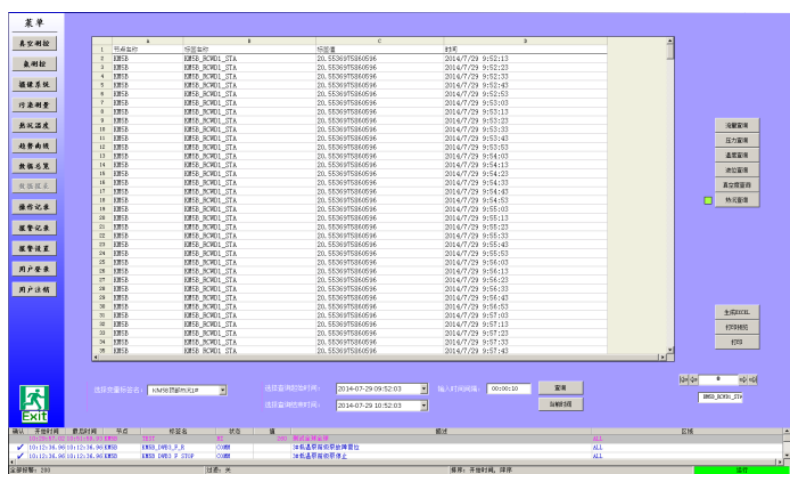

Figure 11 . The schematic of data query

(4) Alarm information: Alarm information of the control system include the real-time alarm information and historical alarm information. The current real-time alarm can be manually processed. The schematic of Alarm information is shown in figure 12.

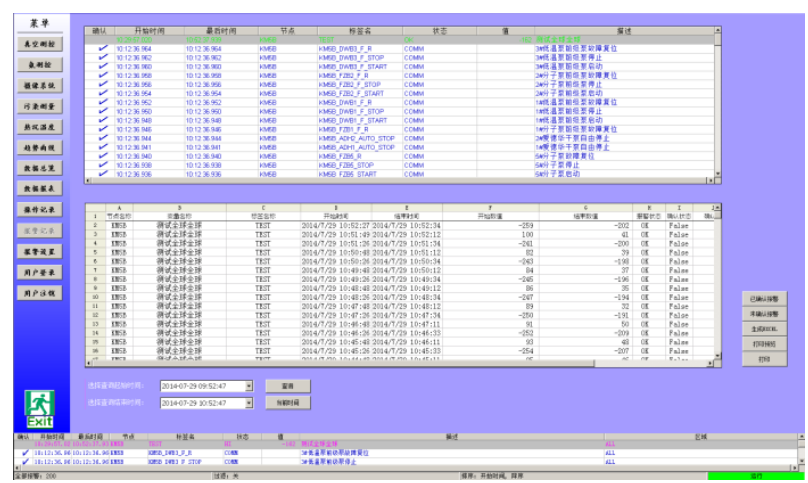

Figure 12. The schematic of alarm information

\section{Conclusion}

This paper introduces a large-scale near space plasma vacuum environment imitation technique. Through calculation and analysis, this paper provides a design scheme required for vacuum working environment. The negative pressure vessel system, vacuum subsystem, vacuum system is introduced in detail.

The finite element analysis and numerical calculation of the mechanical structure and the vacuum capacity verify the feasibility of the design. The indicators of the system proposed in this paper are qualified. It can effectively reduce the influence of wall effect in the research of space plasma.

\section{References}

1. K. Kuriki, H. Kuninaka,. Numerical analysis of interaction of a high-Voltage solar array with ionospheric plasma. $J$ Spacecr. Rockets, 24(6):512-517(2015)

2. M. Cho, D.E. Hastings. Dielectric Charging Processes and Arcing Rates of high Voltage Solar Array, J Spacecr. Rockets, 28(28):698-706(1991)

3. H. Thiemann, R.W. Schunk. Computer experiments on arcing processes as observed in ground tests. $J$ Spacecr. Rockets,31(31):929-936(2015)

4. H. Thiemann, K. Bogus. High-Voltage solar cell modules in simulated low-earth-orbit plasma. J Spacecr. Rockets, 25(4):278-285(2015)

5. Upschule B L et al. Arcing of Negatively Biased Solar Cells in Low Earth Orbit. AIAA 92-0578

6. M.R. Carruth et al. Experimental Studies on Spacecraft Arcing. J Spacecr. Rockets,30(3):323-327(2015)

7. D.E. Hastings, M. Cho, H. Kuninaka. Arcing rates for high voltage solar arrays: theory, experiment and predictions. $J$ Spacecr. Rockets, 29(4):538-554(1992)

8. Quin S, Windra G E O. Use of Stress-Strengh Model in Determination of Safety Factor Pressure Vessel Design[J]. Journal of Pressure Vessel Technology, 118(1):27-32(1996)

9. I. Elishakoff. Essay on Uncertainties in Elastic and Viscoelastic Structures: From A. M. Freudenthal's Crutucusms to Modern Convex Modeling[J]. Computers \& Strultures, 56(6):871-895(1995)

10. Y.M. Zhang, Q.L. Liu. Reliability-Based Design of Automobile Components[J]. Proceedings of the Institution 
of Mechanical Engineers Part D, Journal of Automobile Engineering, 216(6):455-471(2002)

11. B. Huang. Space environment test facility for manned spacecraft[J]. CSST, 22(3): 1-3(2002)

12. Lee Jin,N.S. Harris,N. Tenwick, et a1. Modern vacuum practice[M]. 3rdedition. BOC Edwards. 201-266(2000)

13. J. Tong,L. Sun,R. Jia,Y. Liu, et al . Experimental Techniques in Simulated Space Plasma Environment on Ground [J]. Chinese Journal of Vacuum Science and Technology, 28(3):203-207(2008)

14. M. Ma,T. Chen,J. Lei, et al. Design of Large-Sized Space
Plasma Simulation System for Low Earth Orbit Environment[J]. Chinese Journal of Vacuum Science and Technology, 36(6):680-685(2006)

15. Cho M, Shiraishi K. Laboratory Experiments on Mitigation a-gainst Arcing on High Voltage Solar Array in Simulated LEO Plasma Environment. AIAA2002-0629

16. Tribble A C. Low Earth Orbit Plasma Effect on Spacecraft, A-IAA93-0614

17. Hastings D E. A review of plasma interactions with spaceraft in low earth orbit. J. Geophys. Res, 100(A8):14457-14483(1995) 